ERRATUM

doi:10.1038/nature 15531

\title{
Erratum: Mechanism of phospho-ubiquitin-induced \\ PARKIN activation
}

Tobias Wauer, Michal Simicek, Alexander Schubert

\& David Komander

Nature 524, 370-374 (2015); doi:10.1038/nature14879

The print and PDF versions of this Letter are correct, but the wrong HTML versions of Figs 1-4 and ED Figs 1-10 were used initially, owing to an in-house error; these have been corrected. 